Advanced Computing: An International Journal ( ACIJ ), Vol.3, No.3, May 2012

\title{
DEVELOPMENT OF GROWTH MODELS FOR ELECTRONICS HARDWARE AND SOFTWARE COMPONENTS
}

\author{
Sarojamma B. ${ }^{1}$, Venkatramana Reddy S. ${ }^{2}$, Suresh Kumar Reddy K. ${ }^{3}$ and Hari \\ Mallikarjuna Reddy B. ${ }^{4}$ \\ 1\&4 : Department of Statistics, 2: Department of Physics, 3: Department of Computer \\ Science \\ Sri Venkateswara University, Tirupati - 517 502, Andhra Pradesh, India \\ *drsvreddy123@gmail. com
}

\begin{abstract}
Now a days information technology plays an important role in all fields of sciences, arts and commerce, management and medicine. Up to date information on each and every topic is utilized everybody in all fields through internet. India is investing money in crores in production of software and hardware. Forecast is playing an important role in future prediction. In this paper we develop the growth models for electronic hardware and software components. Suitable Polynomial equations with time ' $t$ ' (independent variable) and time series value ' $y_{i}$ ' (dependent variable) for software production, hardware production and total production are fitted. Coefficient of determination $\left(R^{2}\right)$ measures the percentage variation in the dependent variable, that is accounted by the independent variable.
\end{abstract}

\section{KEYWORDS}

Forecast, Growth models, Software, Hardware \& Coefficient of determination.

\section{INTRODUCTION}

A comparative study of challenges in integrating open source software and inner source software by klaas-jan Stol et al[1], in their paper has an objective to shed light on challenges related to building products with components that have been developed within an inner source development environment. They followed an initial systematic literature review to generate seed category data constructs, and performed an in-depth exploratory case study in an organization that has a significant track record in the implementation of inner source. Data was gathered through semi structured interviews with participants from a range of divisions across the organization. Interviews were transcribed and analyzed using qualitative data analysis techniques.

Reza Meimandi parizi et al [2] studied on "empirical evaluation of the fault detection effectiveness and test effort efficiency of the automated AOP (aspect-oriented programming) testing approaches". In their paper using mutation analysis and examined four existing automated AOP testing approaches i.e. Wrasp [3], Aspectra [4], Raspect [5] and EAT [6]. P.Hu et al [7] studied about "An empirical comparison between direct and indirect test results checking approaches". An empirical study of the robustness of windows NT applications using random testing was done by J.E. Forrester et al [8].

Now a days forecasting plays an important role not only in weather conditions, but also to the business, economics and so many other fields. Forecasting is important to planning better future. In this paper we are fitted a Polynomial equation for forecasting production of Electronics and IT in India. 


\section{Methodology}

In this paper our main objective is to forecasts how many crores that India's future production in hardware, software and total. Before going production to forecast, we have to fit the suitable and appropriate models for the above three variables. Models are tested using coefficient of determination i.e., ' $\mathrm{R}^{2 \text { ' }}$ criterion. The data how we are using the forecasts in time series data. If our data looks as follows:

$$
\begin{array}{ll}
t_{i}: t_{1} & t_{2} \ldots \ldots \ldots \ldots t_{n} \\
y_{i}: y_{1} & y_{2} \ldots \ldots \ldots \ldots y_{n}
\end{array}
$$

Here $t$ is time (independent variable) and

$\mathrm{y}_{\mathrm{i}}$ is time series value.

The suitable equation, dependent variable fitted for the given data is

$$
y_{i}=A t_{i}^{3}+B t_{i}^{2}+C t_{i}+D
$$

i.e. 3 degree polynomial for time ' $t_{i}$ '. The above equation tells about the values of time series value ' $y_{i}$ ' according to change in time ' $t_{i}$ '.

If $\mathrm{A}, \mathrm{B}, \mathrm{C}$ and $\mathrm{D}$ are constants estimated by the Principle of least squares and the fitted equation is

$\hat{y}_{i}=\hat{A} t_{i}^{3}+\hat{B} t_{i}^{2}+\hat{C} t_{i}+\hat{D}$

Difference between the actual time series value and estimated time series value is the error.

$$
y_{i}-\hat{y}_{i}=y_{i}-\left(\hat{A} t_{i}^{3}-\hat{B} t_{i}^{2}-\hat{C} t_{i}-\hat{D}\right)
$$

Error sum of square is obtained by squaring the error values and then total all the squares,

$$
S=\sum_{i=1}^{n}\left(y_{i}-\hat{A} t_{i}^{3}-\hat{B} t_{i}^{2}-\hat{C} t_{i}-\hat{D}\right)^{2}
$$

To minimize 'S', eq. (2) is partially differentiated with respect to A, B, C, D and we equate all its derivatives to zero, and we get four normal equations as follows:

Differentiate the eq. (2) with respect to A and equate to zero, then we get

$$
\begin{aligned}
& \frac{\partial S}{\partial A}=\frac{\partial}{\partial A} \sum_{i=1}^{n}\left(y_{i}-\hat{A} t_{i}^{3}-\hat{B} t_{i}^{2}-\hat{C} t_{i}-\hat{D}\right)^{2} \\
& =2 \sum_{i=1}^{n}\left(y_{i}-\hat{A} t_{i}^{3}-\hat{B} t_{i}^{2}-\hat{C} t_{i}-\widehat{D}\right) \cdot\left(-t_{i}^{3}\right)=0 \\
& \sum_{i=1}^{n}\left(y_{i}-\hat{A} t_{i}^{3}-\hat{B} t_{i}^{2}-\hat{C} t_{i}-\hat{D}\right) t_{i}^{3}=0 \\
& \sum_{i=1}^{n} y_{i} t_{i}^{3}-\hat{A} \sum_{i=1}^{n} t_{i}^{6}-\hat{B} \sum_{i=1}^{n} t_{i}^{5}-\hat{C} \sum_{i=1}^{n} t_{i}^{4}-\hat{D} \sum_{i=1}^{n} t_{i}^{3}=0 \\
& \sum_{i=1}^{n} y_{i} t_{i}^{3}=\hat{A} \sum_{i=1}^{n} t_{i}^{6}+\hat{B} \sum_{i=1}^{n} t_{i}^{5}+\hat{C} \sum_{i=1}^{n} t_{i}^{4}+\hat{D} \sum_{i=1}^{n} t_{i}^{3}
\end{aligned}
$$

Differentiate the equation (2) with respect to B and equate it to zero, we get

$$
\begin{aligned}
& \begin{aligned}
\frac{\partial S}{\partial B} & =\frac{\partial}{\partial B} \sum_{i=1}^{n}\left(y_{i}-\hat{A} t_{i}^{3}-\hat{B} t_{i}^{2}-\hat{C} t_{i}-\hat{D}\right)^{2} \\
& =2 \sum_{i=1}^{n}\left(y_{i}-\hat{A} t_{i}^{3}-\hat{B} t_{i}^{2}-\hat{C} t_{i}-\hat{D}\right) \cdot\left(-t_{i}^{2}\right)=0
\end{aligned} \\
& \sum_{i=1}^{n}\left(y_{i}-\hat{A} t_{i}^{3}-\hat{B} t_{i}^{2}-\hat{C} t_{i}-\hat{D}\right) t_{i}^{2}=0
\end{aligned}
$$




$$
\begin{aligned}
& \sum_{i=1}^{n} y_{i} t_{i}^{2}-\hat{A} \sum_{i=1}^{n} t_{i}^{5}-\hat{B} \sum_{i=1}^{n} t_{i}^{4}-\hat{C} \sum_{i=1}^{n} t_{i}^{3}-\hat{D} \sum_{i=1}^{n} t_{i}^{2}=0 \\
& \sum_{i=1}^{n} y_{i} t_{i}^{2}=\hat{A} \sum_{i=1}^{n} t_{i}^{5}+\hat{B} \sum_{i=1}^{n} t_{i}^{4}+\hat{C} \sum_{i=1}^{n} t_{i}^{3}+\hat{D} \sum_{i=1}^{n} t_{i}^{2}
\end{aligned}
$$

Differentiate the equation (2) with respect to $\mathrm{C}$ and equate it to zero, we get

$$
\begin{aligned}
& \frac{\partial S}{\partial C}=\frac{\partial}{\partial C} \sum_{i=1}^{n}\left(y_{i}-\hat{A} t_{i}^{3}-\hat{B} t_{i}^{2}-\hat{C} t_{i}-\hat{D}\right)^{2} \\
& =2 \sum_{i=1}^{n}\left(y_{i}-\hat{A} t_{i}^{3}-\hat{B} t_{i}^{2}-\hat{C} t_{i}-\widehat{D}\right) \cdot\left(-t_{i}\right)=0 \\
& \sum_{i=1}^{n} y_{i} t_{i}-\hat{A} \sum_{t=1}^{n} t_{i}^{4}-\hat{B} \sum_{t=1}^{n} t_{i}^{3}-\hat{C} \sum_{t=1}^{n} t_{i}^{2}-\hat{D} \sum_{t=1}^{n} t_{i}=0 \\
& \sum_{i=1}^{n} y_{i} t_{i}=\hat{A} \sum_{i=1}^{n} t_{i}^{4}+\hat{B} \sum_{i=1}^{n} t_{i}^{3}+\hat{C} \sum_{i=1}^{n} t_{i}^{2}+\hat{D} \sum_{i=1}^{n} t_{i}
\end{aligned}
$$

Differentiate the equation (2) with respect to D and equate it to zero, we get

$$
\begin{aligned}
& \frac{\partial S}{\partial D}=\frac{\partial}{\partial D} \sum_{i=1}^{n}\left(y_{i}-\hat{A} t_{i}^{3}-\hat{B} t_{i}^{2}-\hat{C} t_{i}-\hat{D}\right)^{2} \\
&=2 \sum_{i=1}^{n}\left(y_{i}-\hat{A} t_{i}^{3}-\hat{B} t_{i}^{2}-\hat{C} t_{i}-\widehat{D}\right) \cdot(-1)=0 \\
& \sum_{i=1}^{n} y_{i}-\hat{A} \sum_{i=1}^{n} t_{i}^{3}-\hat{B} \sum_{i=1}^{n} t_{i}^{2}-\hat{C} \sum_{i=1}^{n} y_{i}-\widehat{D} \sum_{i=1}^{n} 1=0 \\
& \sum_{i=1}^{n} y_{i}-\hat{A} \sum_{i=1}^{n} t_{i}^{3}-\hat{B} \sum_{i=1}^{n} t_{i}^{2}-\hat{C} \sum_{i=1}^{n} t_{i}-n \hat{D}=0 \\
& \sum_{i=1}^{n} y_{i}=\hat{A} \sum_{i=1}^{n} t_{i}^{3}+\hat{B} \sum_{i=1}^{n} t_{i}^{2}+\hat{C} \sum_{i=1}^{n} t_{i}+n \hat{D}
\end{aligned}
$$

By solving the above four normal equations (3), (4), (5) and (6), we obtain the constants A, B, C and D.

By change time $\mathrm{t}_{\mathrm{i}}$ to ' $\mathrm{T}_{\mathrm{i}}$ ', by changing origin and scale equations $\sum_{i=1}^{n} T_{i}=0, \sum_{i=1}^{n} T_{i}^{3}=0$ and $\sum_{i=1}^{n} T_{i}^{5}=0$ and the normal equations becomes

$$
\begin{aligned}
& \sum_{i=1}^{n} y_{i} T_{i}^{3}=\hat{A} \sum_{i=1}^{n} T_{i}^{6}+\hat{C} \sum_{i=1}^{n} T_{i}^{4} \\
& \sum_{i=1}^{n} y_{i} T_{i}^{2}=\hat{B} \sum_{i=1}^{n} T_{i}^{4}+\hat{D} \sum_{i=1}^{n} T_{i}^{2} \\
& \sum_{i=1}^{n} y_{i} T_{i}=\hat{A} \sum_{i=1}^{n} T_{i}^{4}+\hat{C} \sum_{i=1}^{n} T_{i}^{2} \\
& \sum_{i=1}^{n} y_{i} T_{i}=\hat{B} \sum_{i=1}^{n} T_{i}^{2}+n D
\end{aligned}
$$

By solving equations (7) and (9), we get constant $\hat{A}$ and $\hat{C}$.

Multiply eq. (7) with $\frac{\sum_{i=1}^{n} T_{i}^{4}}{\sum_{i=1}^{n} T_{i}^{6}}$, wet get 
$\sum_{i=1}^{n} y_{i} T_{i}^{3} \frac{\sum_{i=1}^{n} T_{i}^{4}}{\sum_{i=1}^{n} T_{i}^{6}}=\hat{A} \sum_{i=1}^{n} T_{i}^{4}+\hat{C} \frac{\left(\sum_{i=1}^{n} T_{i}^{4}\right)^{2}}{\sum_{i=1}^{n} T_{i}^{6}}$

Subtracting equation (9) from (11), we get

$$
\begin{aligned}
& \sum_{i=1}^{n} y_{i} T_{i}^{3} \frac{\sum_{i=1}^{n} T_{i}^{4}}{\sum_{i=1}^{n} T_{i}^{6}}-\sum_{i=1}^{n} y_{i} T_{i}=\hat{C}\left[\frac{\left(\sum_{i=1}^{n} T_{i}^{4}\right)^{2}}{\sum_{i=1}^{n} T_{i}^{6}}-\sum_{i=1}^{n} y_{i} T_{i}\right] \\
& \hat{C}=\frac{\sum_{i=1}^{n} y_{i} T_{i}^{3} \frac{\sum_{i=1}^{n} T_{i}^{4}}{\sum_{i=1}^{n} T_{i}^{6}}-\sum_{i=1}^{n} y_{i} T_{i}}{\frac{\left(\sum_{i=1}^{n} T_{i}^{4}\right)^{2}}{\sum_{i=1}^{n} T_{i}^{6}}-\sum_{i=1}^{n} T_{i}^{2}}
\end{aligned}
$$

Substituting $\hat{C}$ value in equation (9), we get $\hat{A}$

$$
\frac{\sum_{i=1}^{n} y_{i} T_{i}^{3} \frac{\sum_{i=1}^{n} T_{i}^{4}}{\sum_{i=1}^{n} T_{i}^{6}}-\sum_{i=1}^{n} y_{i} T_{i}}{\frac{\left(\sum_{i=1}^{n} T_{i}^{4}\right)^{2}}{\sum_{i=1}^{n} T_{i}^{6}}-\sum_{i=1}^{n} T_{i}^{2}} T_{i}^{2}
$$$$
\sum_{i=1}^{n} y_{i} T_{i}-\sum_{i=1}^{n} T_{i}^{2} \frac{\sum_{i=1}^{n} y_{i} T_{i}^{3} \frac{\sum_{i=1}^{n} T_{i}^{4}}{\sum_{i=1}^{n} T_{i}^{6}}-\sum_{i=1}^{n} y_{i} T_{i}}{\frac{\left(\sum_{i=1}^{n} T_{i}^{4}\right)^{2}}{\sum_{i=1}^{n} T_{i}^{6}}-\sum_{i=1}^{n} T_{i}^{2}}=\hat{A} \sum_{i=1}^{n} T_{i}^{4}=\mathrm{N}_{1}
$$

$$
D_{1}=\sum_{i=1}^{n} T_{i}^{4}
$$

Equation (14) in (13), we get

$$
N_{1}=\hat{A} D_{1}
$$




$$
\hat{A}=\frac{N_{1}}{D_{1}}
$$

Solving equations (8) and (10), we obtain $\hat{B}$ and $\hat{C}$.

Multiply eq. (10) with $\frac{\sum_{i=1}^{n} T_{i}^{2}}{n}$ then we get,

$$
\sum_{i=1}^{n} y_{i} T_{i} \frac{\sum_{i=1}^{n} T_{i}^{2}}{n}=\hat{B} \frac{\left(\sum_{i=1}^{n} T_{i}^{2}\right)^{2}}{n}+\sum_{i=1}^{n} T_{i}^{2} \hat{D}
$$

Subtracting equation (8) from equation (15), we get

$$
=\sum_{i=1}^{n} y_{i} T_{i} \frac{\sum_{i=1}^{n} T_{i}^{2}}{n}-\sum_{i=1}^{n} y_{i} T_{i}^{2}=\hat{B}\left(\frac{\left(\sum_{i=1}^{n} T_{i}^{2}\right)^{2}}{n}-\sum_{i=1}^{n} T_{i}^{4}\right) \hat{B}=\frac{\sum_{i=1}^{n} y_{i} T_{i} \frac{\sum_{i=1}^{n} T_{i}^{2}}{n}-\sum_{i=1}^{n} y_{i} T_{i}^{2}}{\frac{\left(\sum_{i=1}^{n} T_{i}^{2}\right)^{2}}{n}-\sum_{i=1}^{n} T_{i}^{4}}
$$

Substituting equation (16) in equation (10), we get

$$
\begin{aligned}
& \sum_{i=1}^{n} y_{i} T_{i}=\frac{\sum_{i=1}^{n} y_{i} T_{i} \frac{\sum_{i=1}^{n} T_{i}^{2}}{n}-\sum_{i=1}^{n} y_{i} T_{i}^{2}}{\frac{\left(\sum_{i=1}^{n} T_{i}^{2}\right)^{2}}{n}-\sum_{i=1}^{n} T_{i}^{4}} \cdot \sum_{i=1}^{n} T_{i}^{2}+n D \\
& \sum_{i=1}^{n} y_{i} T_{i}-\frac{\sum_{i=1}^{n} y_{i} T_{i} \frac{\sum_{i=1}^{n} T_{i}^{2}}{n}-\sum_{i=1}^{n} y_{i} T_{i}^{2}}{\left(\sum_{i=1}^{n} T_{i}^{2}\right)^{2}}-\sum_{i=1}^{n} T_{i}^{2}=n D \\
& N_{2}=n D \\
& D=\frac{N_{2}}{n}
\end{aligned}
$$

The fitted polynomial equation is

$$
\hat{y}=\hat{A} T^{3}+\hat{B} T^{2}+\hat{C} T+D
$$

\subsection{Coefficient of determination $\left(\mathbf{R}^{2}\right)$}

Correlation coefficient between two variables measures the linear relationship between them and indicates the amount of variation of one variable which is associated with or is accounted for by another variable. Square of correlation coefficient is coefficient of determination $\mathrm{R}^{2}$. In other words the coefficient of determination gives the percentage variation in the dependent variable that is accounted for by the independent variable. Coefficient of determination gives the ratio of the explained variance to total variance. 
$\mathrm{R}^{2}$ (Coefficient of determination) $=\frac{\text { Explained variance }}{\text { Total variance }}$

\subsection{Empirical investigations}

A suitable and appropriate model fitted to time series data of software, hardware and total production by India. The appropriate model for three time series data is

$\hat{y}=A t_{i}^{3}+B t_{i}^{2}+C t_{i}+D$

where $t_{i}$ is Time at $\mathrm{i}^{\text {th }}$ time period

$y_{i}$ is Time series value at $\mathrm{i}^{\text {th }}$ time period

$\mathrm{A}, \mathrm{B}, \mathrm{C}$ and $\mathrm{D}$ are constants

The above equation is the third degree polynomial growth equation.

Electronics production data (hardware and software) in each year is given in Table-1[9]. The hardware includes consumer electronics, industrial electronics, computer hard ware, communication and broadcast equipment, strategic electronics and electronic components. Software includes software for exports and domestic software, total production is both hardware and software.

Table 1

\begin{tabular}{|c|c|c|c|}
\hline Time & Software & Hardware & Total production \\
\hline 1999 & 27750 & 23000 & 50750 \\
\hline 2000 & 30880 & 35800 & 66680 \\
\hline 2001 & 32150 & 44600 & 76750 \\
\hline 2002 & 36800 & 56000 & 92800 \\
\hline 2003 & 42700 & 70500 & 113200 \\
\hline 2004 & 49800 & 95500 & 145300 \\
\hline 2005 & 54500 & 124000 & 178500 \\
\hline 2006 & 64400 & 167175 & 231575 \\
\hline 2007 & 79800 & 203060 & 282860 \\
\hline 2008 & 94060 & 259260 & 353300 \\
\hline 2009 & 107370 & 297400 & 404770 \\
\hline
\end{tabular}

\subsection{Software production}

The fitted equation for the software production data is

$\mathrm{y}=31.31 \mathrm{~T}^{3}+234 \mathrm{~T}^{2}+1076 \mathrm{~T}+26754$

Coefficient of determination $R^{2}$ is 0.997 i.e. $99.7 \%$ of the time series value (y) and has been explained by the time ' $t$ ' and the remaining $0.3 \%$ of the variation is due to other factors.

\subsection{Hardware production}

The fitted equation for the hardware export data is

$\mathrm{y}=19.24 \mathrm{~T}^{3}+2315 \mathrm{~T}^{2}-2782 \mathrm{~T}+27665$

Coefficient of determination $R^{2}$ is 0.997 i.e. $99.7 \%$ of time series value (y) and has been explained by the time ' $t$ ' and the remaining $0.3 \%$ of the variation is due to other factors.

\subsection{Total production}

The fitted equation for the total production data is $\mathrm{y}=50.59 \mathrm{~T}^{3}+2549 \mathrm{~T}^{2}-1702 \mathrm{~T}+54415$ 
Coefficient of determination $R^{2}$ is 0.998 i.e. $99.8 \%$ of time series values (y) and has been explained by the time ' $\mathrm{t}$ ' and the remaining $0.2 \%$ of the variation is due to other factors.

Plots are drawn by taking time ' $\mathrm{t}$ ' on $\mathrm{x}$-axis and time series value ' $\mathrm{y}$ ' on $\mathrm{y}$-axis for software production using equation (18), hardware production using equation (19) and total production using equation (20). The corresponding curves are shown in Figure 1, Figure 2 and Figure 3 respectively for both actual data and forecast data. The curves for original time series values and forecast time series values are coincide.

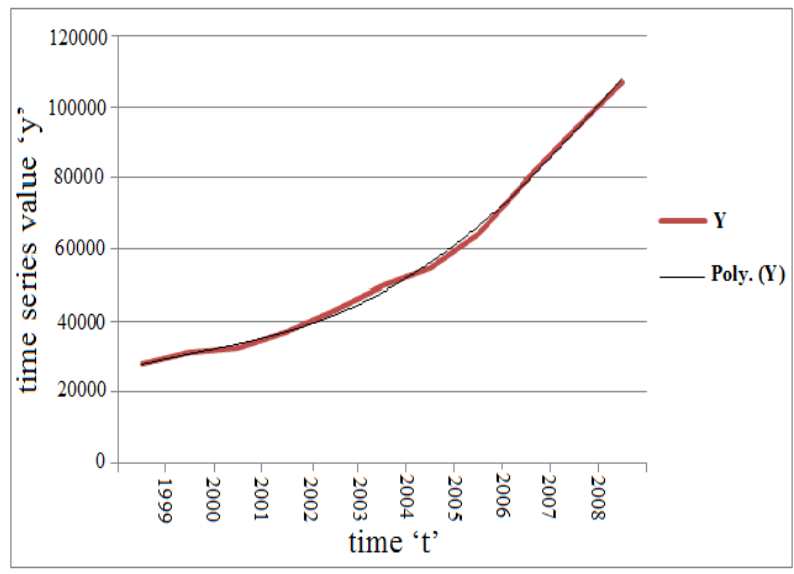

Figure 1. Software Production for actual data and forecast data.

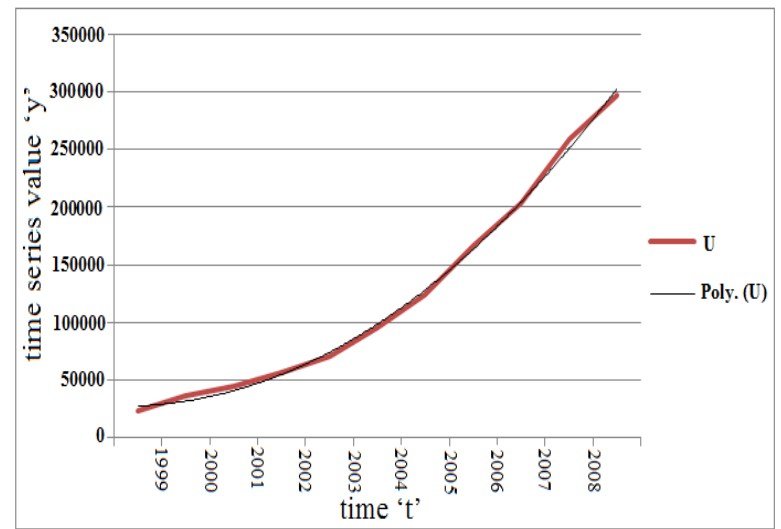

Figure 2. Hardware Production for actual data and forecast data.

Table -2 contains 4 columns, first column tells about the time ' $t$ ' in years, second column tells about percentage growth of software exports, third and fourth columns gives the information regarding the percentage growth of hardware and total exports. 
Advanced Computing: An International Journal ( ACIJ ), Vol.3, No.3, May 2012

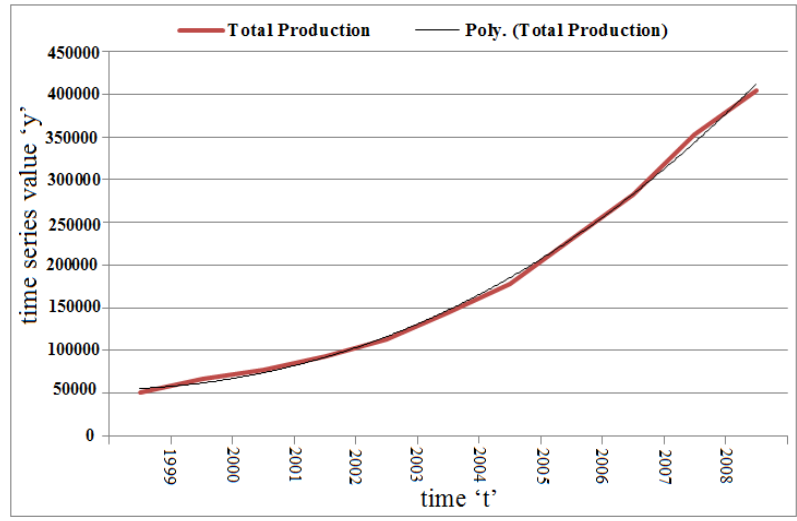

Figure 3. Total Production for actual data and forecast data.

Table 2

\begin{tabular}{|c|c|c|c|}
\hline Time & Software & Hardware & Total \\
\hline 1999 & ----- & ----- & ----- \\
\hline 2000 & 0.112793 & 0.56 & 0.31 \\
\hline 2001 & 0.04 & 0.24 & 0.15 \\
\hline 2002 & 0.15 & 0.25 & 0.21 \\
\hline 2003 & 0.16 & 0.26 & 0.22 \\
\hline 2004 & 0.16 & 0.35 & 0.28 \\
\hline 2005 & 0.10 & 0.30 & 0.23 \\
\hline 2006 & 0.18 & 0.25 & 0.30 \\
\hline 2007 & 0.24 & 0.25 & 0.22 \\
\hline 2008 & 0.18 & 0.28 & 0.25 \\
\hline 2009 & 0.14 & 0.15 & 0.16 \\
\hline Average & 0.15 & 0.30 & 0.23 \\
\hline
\end{tabular}

Table-3 gives the information for the production, fitted polynomial equation, coefficient of determination and average growth rate per year.

Table 3

\begin{tabular}{|c|c|c|c|}
\hline $\begin{array}{c}\text { Type of } \\
\text { production }\end{array}$ & Fitted Polynomial Equation & $\begin{array}{c}\text { Coefficient of } \\
\text { Determination } \\
\left(\mathbf{R}^{2}\right)\end{array}$ & $\begin{array}{c}\text { Average } \\
\text { growth rate } \\
\text { per Annum }\end{array}$ \\
\hline Software & $31.31 \mathrm{~T}^{3}+234 \mathrm{~T}^{2}+1076 \mathrm{~T}+26754$ & 0.997 & $15 \%$ \\
\hline Hardware & $19.24 \mathrm{~T}^{3}+2315 \mathrm{~T}^{2}-2782 \mathrm{~T}+27665$ & 0.997 & $30 \%$ \\
\hline Total & $50.59 \mathrm{~T}^{3}+2549 \mathrm{~T}^{2}-1702 \mathrm{~T}+54415$ & 0.998 & $23 \%$ \\
\hline
\end{tabular}

On an average $15 \%$ growth rate is recorded for software, $30 \%$ growth rate for hardware and $23 \%$ growth rate for total exports.

\section{Conclusions}

The growth models for hardware, software and total production are developed. The fitted equation (18) for software exports of India with coefficient of determination $\left(R^{2}\right)$ is 0.997 and average growth rate per annum is 15 percent. The fitted equation (19) for hardware exports of 
India with coefficient of determination $\left(\mathrm{R}^{2}\right)$ is 0.997 and 33 percent of average growth rate is showing per annum. The fitted equation (20) for total exports in India with coefficient of determination $\left(\mathrm{R}^{2}\right)$ is 0.998 and 23 percent growth is observed on an average per annum.

\section{REFERENCES}

[1] Klaas-Jan Stol, Muhammad Ali Babar, Paris Avgeriou and Brian Fitzgerald, (2011) "A comparative study of challenges in integrating open source software and inner source software", Journal of information and software technology, vol. 53, pp. 1319-1336.

[2] Reza Meimandi Parizi, Abdul azim Abdul Ghani, Rusli Abdullah and Rodziah Atan, (2011) "Empirical evaluation of the fault detection effective and test effort efficiency of the automated AOP testing approaches", Journal information and software technology, vol. 53, pp. 1062-1083.

[3] T. Xie, J. Zhao, D. Marinov and D. Notkin, (2005) "Automated test generation for aspect programs", Proc. $1^{\text {st }}$ workshop on testing Aspect-Oriented programs, pp.1-6.

[4] T. Xie and J. Zhao, (2006) "A framework and tool supports for generating test inputs of aspect programs", Proc. $5^{\text {th }}$ international conference on Aspect-Oriented Software development, ACM, pp.190-201.

[5] T. Xie, J. Zhao, D. Marinov and D. Notkin, (2006) "Detecting redundant unit tests for Aspect programs", Proc. $17^{\text {th }}$ international symposium on software reliability Engineering, IEEE computer society, pp.179-190.

[6] M. Harman, F. Islam, T. Xie and S. Wrappler, (2009) "Automated test data generation for aspectoriented programs", Proc. international conference on aspect-oriented software development, ACM, Chrlottesville, Virginia,USA, pp.185-196.

[7] P. Hu, Z. Zhang, W.K. Chan and T.H. Tse, (2006) "An empirical comparison between direct and indirect test result checking approaches", Proc. $3^{\text {rd }}$ international workshop on software Quality Assurance, ACM, Portland, Oregon, pp.6-13.

[8] J.E. Forrester and B.P. Miller, (2000) "An empirical study of the roubustness of windows NT applications using random testing”, Proc. $4^{\text {th }}$ conference on USENIX windows Systems Symposium, pp.59-68.

[9] Information Technology Annual report (2009-2010) website. [Online]. Available: http://www.mit.gov.in/sites/upload_files/dit/files/annualreport2009-10_0.pdf

\section{Authors}

Dr. S. Venkatramana Reddy received M.Phil. and Ph.D. degrees in Physics in 1996, 2001 respectively from Sri Venkateswara University, Tirupati, Andhra Pradesh, India. He taught the subjects Semiconductor Devices \& Circuits, Analog and Digital Electronics, 8051 Microcontrollers \& Applications, Embedded Systems using PIC microcontrollers, Advanced Microprocessors \& Microcomputers, Electronic Instrumentation, Industrial Control Electronics, Analog \& Digital Communication, Microwave and Satellite communication, Optical Fiber Communications, Advanced Communication Systems, Mathematical Physics, etc. to the students of M.Sc. Electronics/ M.Tech. Energy Management/ M.Sc. Physics/ 5yr Integrated M.Sc. in Physics since from the academic year 1995-1996. He has been actively involved in designing M.Sc. Electronics Course in the University. He has

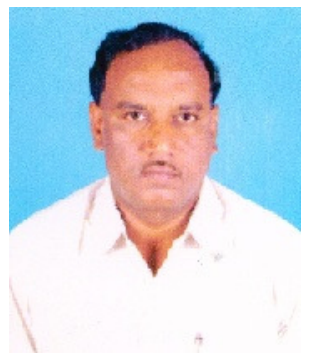
published more than 27 research papers in internationally reputed Journals and presented 33 papers at National/International Conferences/Symposia. He attended seven Electronics oriented Training Courses/Workshops/National Schools. He is Fellow, Institution of Electronics and Telecommunication Engineers, New Delhi, Senior member in International Association of Computer Science and Information Technology, Life member (LM) in many Professional 
Bodies like the Instrument Society of India, Bangalore, Semiconductor Society (India), New Delhi, Indian Physics Association, BARC, Mumbai, Indian Association of Physics Teachers, Kanpur, Uttar Pradesh and Member in International Association of Engineers. He organised a one day workshop on Satellite Navigation Systems - their application to Aviation and atmospheric science. At present he is working as An Assistant Professor in the Department of Physics, S.V. University, Tirupati.

Dr. B. Sarojamma received M.Sc. and Ph.D. degrees in Statistics in 2000, 2006 respectively from Sri Venkateswara University, Tirupati, Andhra Pradesh, India. She taught the subjects Time series and Forecasting, Operations Research, Operations Research for Management, Advanced Operations Research, Distribution Theory, Sampling techniques and Biostatistics, etc. to the students of M.Sc. Statistics, Applied Statistics, and M.Sc. Bio-technology. She has given several Invited lectures in National/International workshops/conferences. She has published 13 research papers in internationally reputed Journals and presented more than 20 papers at National/International Conferences/Symposia. She is Life member (LM) in many Professional Bodies like Indian Society for Probability and Statistics (ISPS), Andhra Pradesh Society for Mathematical Sciences, Hyderabad, International Indian Statistics Association (IISA), Cochin, Indian Mathematical Society (IMS), Aurangabad and Indian Statistical Association (ISA), Pune. At present she is working as An Assistant Professor in the Department of Statistics, S.V. University, Tirupati.

Dr. K. Suresh Kumar Reddy received M.Sc. and Ph.D. degrees in Computer Science in 2000, 2011 respectively from Sri Venkateswara University, Tirupati, Andhra Pradesh, India. He taught the subjects related to Computer Science in various Departments such as Computer Science, M.Sc. Electronics, 5 year M.Sc Integrated Course in Physics, Home Science, Dept, of Education etc., He has published 6 research papers in internationally reputed Journals and presented more than 10 papers at National/International Conferences/Symposia. At present he is working as Academic Consultant, in S.V. University, Tirupati.

Mr. B. Hari Mallikarjuna Reddy received M.Sc. degree in Statistics in 2007 from Sri Venkateswara University, Tirupati, Andhra Pradesh, India. He also submitted Ph.D. in Statistics. He taught Statistics papers in various Departments like M.Sc Statistics, Home Science etc., He has published 5 research papers in internationally reputed Journals and presented more than 15 papers at National/International Conferences/Symposia. At present he is working as Academic Consultant in S.V. University, Tirupati.
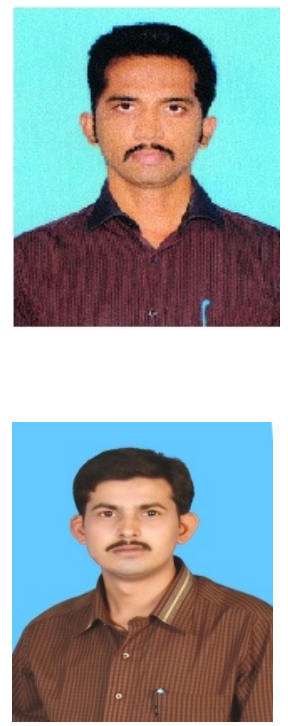